\title{
Identifying Service Needs from the Perspective of Service Providers-A Qualitative Study
}

\author{
Shih-Ying Chien ${ }^{1,2}$, Ming-Chuen Chuang1, I-Ping Chen ${ }^{1}$ \\ ${ }^{1}$ Institute of Applied Arts, National Chiao Tung University, Hsinchu, Taiwan \\ ${ }^{2}$ Institute of East Asian Studies, University of California, Berkeley, USA \\ Email: shihying4768@berkeley.edu, shihying4768@gmail.com
}

How to cite this paper: Chien, S.-Y., Chuang, M.-C. and Chen, I.-P. (2019) Identifying Service Needs from the Perspective of Service Providers-A Qualitative Study. Health, 11, 489-500.

https://doi.org/10.4236/health.2019.115042

Received: April 8, 2019

Accepted: May 13, 2019

Published: May 16, 2019

Copyright $\odot 2019$ by author(s) and Scientific Research Publishing Inc. This work is licensed under the Creative Commons Attribution International License (CC BY 4.0).

http://creativecommons.org/licenses/by/4.0/

\begin{abstract}
The provision of high-quality medical care has become a key challenge in many countries. At the same time, there is a growing awareness that the early detection of chronic diseases can reduce mortality rates. Therefore, it is important to identify service needs which influence to participate in health screening. To date, little research has been conducted into the perspectives of service providers on this topic. In this preliminary study, we aimed to explore the perspectives of service providers who work in a community-based setting in order to identify perceived barriers to chronic disease screening and incentives which can increase participation rates. In-depth interviews were conducted with 10 experienced health service providers from October 2017 to December 2017. Interviewees included physicians and community health representatives who had been working in a community-based health screening service for at least 3 years. The study identified several aspects of health screening services for chronic diseases that are in need of improvement, including a shortage of appropriate health service providers, inadequate attendance promoting measures, a lack of continually accessible and affordable medical services, healthcare professionals who possess inadequate communication skills, and rural-urban health care disparities. Service providers believe that current services do not fulfill community needs. Older adults who live in rural areas experience especially significant screening-related health disparities, which are mainly affected by structural service barriers. To improve the quality of medical services, healthcare screening services should be evaluated according to the perspectives of both participants and service providers.
\end{abstract}

\section{Keywords}

Service Providers, Health Screening, Participation Rates, Service Needs

\section{Introduction}

Chronic diseases are among the most prevalent and costly health problems in 
the world [1]. Indeed, chronic diseases are the leading cause of poor health, disability, and death, and are also the primary source of healthcare expenditures [2]. According to research on chronic diseases by the Centers for Disease Control and Prevention [3], in 2012, approximately 117 million people suffered from one or more chronic health conditions, and that number is growing. There is no doubt that detecting chronic diseases early may lead to better disease control and longer survival times [4].

The burden that chronic diseases pose to national healthcare is increasing rapidly worldwide [5]. Although empirical evidence suggests that screening is effective at reducing the risk of death from chronic diseases [6] [7], morbidity and mortality rates continue to rise, in part due to low health screening participation rates [8]. However, early detection and treatment of chronic diseases are possible, and this leads to a better prognosis. Unfortunately, people often neglect the importance of preventative measures, which can lead to delays in treatment.

Research into the perspectives of service providers is to understand more health services to people who suffer from chronic diseases. Currently, there are very few studies defining service requirements from the perspective of service providers. This type of research is challenging due to the fact that different participants from different ages and cultural backgrounds have different needs. Therefore, perspectives of service providers are increasingly recognized as the key to the redesign of health care processes and services. Since participants come from different age groups, cultures, and educational backgrounds, screening requirements and ideas will differ. Therefore, service providers' perspectives will be considered an essential factor in the design of the service process.

The opinions of service providers have proven to be especially valuable because they understand participants' needs [9] and know what real problem for the health screening service. In the current research, taking account of service quality can result in reduced willingness for people to participate in health screening. In addition, the opinions of healthcare service providers can benefit the design of health screening services, and a better understanding of health screening services can lead to improvements in the quality and effectiveness of medical services. This study, therefore, explores the perspectives of service providers as they pertain to the delivery of health services in four representative townships located in the north of Taiwan (Table 1).

\section{Methods}

We performed a qualitative study in which individual interviews were conducted with service providers according to a grounded theory approach. The grounded theory approach is suitable when exploring new topics for which little knowledge is known [10]. Since understanding service factors and barriers is the first step in motivating people to undergo preventative health screening, this study focused on the perspectives, experiences, and perceptions of service providers who perform health screening for chronic diseases in a community-based setting. Specifically, we conducted interviews in order to understand the following: 
Table 1. Sociodemographic and clinical characteristics of the participants.

\begin{tabular}{ccc}
\hline Districts & Sociodemographic & Clinical characteristics \\
\hline A & Urban area (general population) & General chronic diseases (Metabolism) \\
B, C, D & Rural area (fishmen, miners) & High risk of liver, kidney, and lung diseases \\
\hline
\end{tabular}

- The characteristics of service providers who perform health screening for chronic diseases in community-based settings.

- Avoidable service gaps which frequently cause participants to complain or to refuse to undergo health screening services.

- Incentives and/or services that are currently used and that are effective in motivating participants to continue to use health screening services.

- Service gaps and unmet needs.

\subsection{Interviewees Recruitment}

We conducted semi-structured in-depth interviews with 10 experienced healthcare service providers ( 5 females and 5 males). These individuals comprised nurses, research assistants, community health representatives, and physicians who had been working for a community-based health screening service in Chang Gung Memorial Hospital (CGMH), Taiwan, for at least 3 years. Every interviewee takes separated responsibility in current health screening services.

We used purposive sampling to recruit interviewees from different job positions in order to achieve maximal variation. The program director helped to send emails to invite staffs to take part in this study. We then made appointments with the participants and conducted the in-depth interviews anonymously, so that the interviewees could comfortably discuss and disclose their views without hierarchical influences.

\subsection{Interview Guide and Data Collection}

The study was approved by the Institutional Review Board (IRB) of the Chang Gung Memorial Hospital (CGMH). Face-to-face interviews were conducted from October 2017 to December 2017.

Prior to the start of the interview process, the main researcher explained the study objectives, and interviewees were asked to complete a brief demographic survey and talk about their experiences pertaining to current health screening services. For example, they were asked to describe service gaps they would like to address and/or negative experiences that they have had. Each interview was approximately 1 hour in length, and all interviews were digitally recorded and transcribed for further analysis.

\subsection{Data Analysis}

All interview transcripts were entered into NVivo10 software so that qualitative data could be coded, searched, organized, and compared with the audio recording for accuracy. The code was divided into five categories, which were selected 
based on the experience of service providers. Interviews sought to establish:

- Job descriptions and work experiences of Interviewees

- Participants' behavioral observations

- Challenges associated with service delivery

- Service needs (identified according to service providers perspectives)

During the analysis stage, transcripts were reviewed by members of the research team, who ensured that no key ideas had been overlooked. Primary and recurrent issues within each theme were clustered and identified.

\subsection{Ethical Considerations}

For ethical purposes, the identities of interviewees were kept confidential and anonymous. All interviewees were informed about the purpose and design of the study prior to the start of the interview.

\section{Results}

Findings of the current qualitative study are presented according to the different themes identified during the data analysis. These main themes including: 1) general perspectives of service providers; 2 ) shortage of health care providers; 3 ) lack of recall system; 4) biased registration mechanism 5) rural-urban disparity in health care services; 6) low education level and rurality. Table 2 shows the main themes and supporting quotations.

\subsection{General Perspectives of Service Providers}

The heavy workload borne by health service providers was a common theme in most interviews. This is a critical problem in many countries that must be addressed through innovative strategies such as the use of financial incentives to motivate and retain health service providers. Several interviewees described their work as an arduous and thankless task.

Our screening process is in many instances limited by manpower and location. For example, we have never offered an ultrasound exam, which requires physician availability and a location that can house ultrasound equipment. Therefore, screening participants are often not satisfied with our services. (Interviewee 7)

During the interviews, we were told repeatedly that, due to a shortage of health service providers, currently employed providers are often forced to work long hours and feel overburdened. On the other hand, many interviewees reported that they were contracted employees, which means that their jobs often lack security and suffer from inadequate pay structures. As a result, these health service providers reported feeling burnout and described high turnover rates at their clinic/facility.

Aside from physicians, the vast majority of medical providers are contractors, meaning that we lack job security and salary requirements. This has also caused many of our colleagues to leave in search of more stable, long-term employment. (Interviewee 1) 
Table 2. The main themes and example quotations.

\begin{tabular}{ll}
\hline Main themes & \multicolumn{1}{c}{ Supporting quotes } \\
\hline "Our screening process is in many instances limited by manpower and location." \\
"Aside from physicians, the vast majority of medical providers are contractors, meaning that we \\
lack job security and salary requirements. This has also caused many of our colleagues to leave \\
in search of more stable, long-term employment." \\
"Our program employs temps and interns from various industries who are not familiar with the \\
service workflow of the program, leading to difficulties such as confusing the on-site screening \\
process and giving the wrong instructions." \\
"We spend too much time waiting in line, and the entire process also takes too long."
\end{tabular}

"When we are understaffed, the program coordinator hires interns and temporary staff to make up the difference. However, the temporary staff (including the interns) does not fully understand the program, so we must spend time every week training them and correcting

Shortage of health care providers their mistakes."

"The most common complaint from screening participants is long wait times, which is related to the screening experience. We believe that training for full-time and temporary staff and the entire workflow need improvement."

Lack of recall system

'I believe that most people are unwilling to answer phone calls from an unknown number for fear of being defrauded. At the same time, elderly people residing in rural areas seldom use mobile phones."

"Several residents mentioned the village representative as the reason for their inability to register for and participate in their community's medical screenings: the village representative

Biased registration mechanism limits the screening quota to his friends and family even though it infringes on the rights of other locals."

"Every year we receive complaints by phone about the village representative monopolizing registration mechanics, resulting in service gaps."

"Provided services are usually limited by provider availability and the township screening site. Every year, participants ask if we will provide X-rays, ultrasound, and bone density exams (and often, we cannot)."

Rural-urban disparity in health care services "When residents learn that we cannot provide ultrasounds, they disappointedly tell us that without advanced equipment the screening cannot be called a comprehensive health checkup." "In the course of my work, about half of women have asked why we do not provide pap smears? This is difficult to answer because we do not have a gynecologist."

"On the whole, the participants are mostly elderly living in rural areas. According to my observations, the general education level of the elderly in the rural areas is low, and some of the participants seem to believe that the services we provide are not beneficial and make them feel uncomfortable. For example, most of the elderly only speak the Southern Min dialect, and communication barriers often leave them feeling lost."

Low education level and rurality

"I believe a majority of participants, especially the elderly living in rural areas, trust and depend on us. At the same time, the majority of the elderly population, particularly the illiterate, remains uneducated about health. This is why it is difficult to adhere to the screening process, an indicator that we need additional assistance. Also, the more elderly in the screening area, the more time and manpower."

Our program employs temps and interns from various industries who are not familiar with the service workflow of the program, leading to difficulties such as confusing the on-site screening process and giving the wrong instructions. (Interviewee 3)

Interviewees also indicated that participants frequently complained about wait times.

We spend too much time waiting in line, and the entire process also takes too long. (Interviewee 1) 


\subsection{About Service Gaps}

Service gap 1: Shortage of health care providers

During the interviews, a shortage of healthcare workers was frequently mentioned as a serious problem. Improved job security and a well-designed salary structure could be an effective way to improve the job satisfaction of health care providers.

Interviewees identified a lack of skilled healthcare providers who were trained to perform health screenings as a barrier to effectively addressing chronic diseases. In addition, interviewees noted that, if service providers were knowledgeable about the operation of instruments used during the screening process, then screenings would be more efficient.

When we are understaffed, the program coordinator hires interns and temporary staff to make up the difference. However, the temporary staff (including the interns) does not fully understand the program, so we must spend time every week training them and correcting their mistakes. (Interviewee 4)

The most common complaint from screening participants is long wait times, which is related to the screening experience. We believe that training for full-time and temporary staff and the entire workflow need improvement. (Interviewee 5)

Teamwork is essential in the provision of healthcare services. However, there is often limited knowledge about how to improve camaraderie among the service team, and even less information on how to develop the skills required for teamwork, especially when employees have different backgrounds. Health service providers also have concerns about service quality, including the accurate transfer of information and service process operation.

Service gap 2: Lack of recall system

To increase health screening participation rates, many health service providers provide appointment reminders via phone calls. Unfortunately, the effectiveness of phone reminders is often limited, as not all participants receive the phone call and many participants are reluctant to answer a call from an unknown number.

I believe that most people are unwilling to answer phone calls from an unknown number for fear of being defrauded. At the same time, elderly people residing in rural areas seldom use mobile phones. (Interviewee 7)

Many interviewees mentioned that the participants in this study are senior citizens who live in a rural area, which poses greater challenges to health screening. Such participants typically have less access to health information and medical resources compared to patients in urban areas. As a lack of information was identified as an important barrier to screening, interviewees expressed that novel approaches, such as door-to-door advertising, may reduce access barriers for rural seniors to health screening services.

Service gap 3: Biased registration mechanism

Since health screenings of chronic disease are provided free of charge, the 
maximum number of participants each time is 120 . In this health screening service, the recruiting process is completed by the village representative, who also helps with service promotion and registration. However, many interviewees mentioned that participants felt that the registration process is unfair. Indeed, a commonly-reported problem was that the village representative retained some screening opportunities for his/her own friends and family alone. However, from a medical standpoint, it is best to offer screening services to participants who have been screened before, since many programs seek to track the health status of participants for 5 years. Therefore, a more open and fair registration process which prioritizes previously-screened participants is recommended.

Several residents mentioned the village representative as the reason for their inability to register for and participate in their community's medical screenings: the village representative limits the screening quota to his friends and family even though it infringes on the rights of other locals. He insists on the quota simply because he knows who will or will not vote for him. (Interviewee 2)

Every year we receive complaints by phone about the village representative monopolizing registration mechanism, resulting in service gaps. (Interviewee 8)

One important finding of this research was that willingness to participate in a health screening depends on every detail of service design, such as registration system design, screening process arrangement, etc. Such factors influence willingness to participate in health screening. Furthermore, when patients feel that their rights and interests have been encroached upon, their emotions and willingness to participate in health screening are directly affected, and so are service outcomes.

Service gap 4: Rural-urban disparity in health care services

Many studies have shown that the availability of various healthcare screening services influences an individual's willingness to undergo a screening. In the current study, interviewees indicated that there was little difference in participants' expectation between densely-populated urban areas and sparsely-populated rural areas.

Provided services are usually limited by provider availability and the township screening site. Every year, participants ask if we will provide $X$-rays, ultrasound, and bone density exams (and often, we cannot). (Interviewee 7)

When residents learn that we cannot provide ultrasounds, they disappointedly tell us that without advanced equipment the screening cannot be called a comprehensive health checkup. (Interviewee 10)

In the course of my work, about half of women have asked why we do not provide pap smears. This is difficult to answer because we do not have a gynecologist. (Interviewee 9)

Health screenings can detect medical conditions during the early stages of chronic disease (before symptoms present), and screening equipment plays a 
valuable role in health screening. However, many patients in the studied communities did not seem satisfied with available hospital services because the screening equipment that the hospitals had access to was limited. This lack of availability can also reduce willingness to participate in health screenings. If medical equipment is effective and properly designed, participants can enjoy better care.

\subsection{Low Education Level and Rurality}

Many interviewees reported that education level has a key influence over willingness to participate in health screening. Indeed, low educational levels were identified as barriers to understanding 1) the importance of chronic disease prevention and 2) how to access health services.

I think the low participation rate is related to health cognition and health awareness of the participants. Our job is not only to help people understand chronic diseases, but also to expose them to healthy eating and medical services. (Interviewee 4)

Interviewees also noted that health services currently suffer from a shortage of providers who possess strong communication skills and are able to speak with participants in their own language. Such providers are especially important when treating or screening participants with a lower level of education.

On the whole, the participants are mostly elderly living in rural areas. According to my observations, the general education level of the elderly in the rural areas is low, and some of the participants seem to believe that the services we provide are not beneficial and make them feel uncomfortable. For example, most of the elderly only speak the Southern Min dialect, and communication barriers often leave them feeling lost. (Interviewee 6)

I believe a majority of participants, especially the elderly living in rural areas, trust and depend on us. At the same time, the majority of the elderly population, particularly the illiterate, remains uneducated about health. This is why it is difficult to adhere to the screening process, an indicator that we need additional assistance. Also, the more elderly there are in the screening area, the more time and manpower we will use. (Interviewee 5)

At the end of the interviews, I asked interviewees which issue was the most important priority for immediate improvement. Most of the interviewees responded that health care delivery should be adjusted to help improve perceptions of hospital service quality.

\section{Discussion}

The purpose of this study was to identify service needs according to the perspectives of service providers. We found that service providers 1) felt overwhelmed at work and 2) believed that more qualified service providers should be hired. Most of the interviewees in our study also reported that, like labor shortages and irregular working hours [11] [12], a lack of adequate service provider training 
posed a significant challenge to the delivery of effective healthcare services. In addition, most interviewees in our study noted that they were contract employees and therefore did not have good job security. Prytherch et al. [13] reported that a well-designed salary structure and incentives could encourage service providers to work harder. In other words, benefits can have a great influence on the performance and attitude of service providers.

Most interviewees also reported that they were medical workers as well as research assistants. To ensure that people could fit screenings into their schedule, the service had to be arranged in the weekend. Thus, service providers had to work overtime. Continuously working for a week exhausted made them lose quality time with friends and family. This caused some health service providers chose to leave their jobs.

About half of the interviewees also noted that the provision of quality of healthcare services depends on the whole service system design which includes high-quality screening equipment, service provided and a well-trained team [8] [14] [15]. Specifically, if more professional physicians were available to provide a greater number of healthcare services, health screening rates could be greatly increased. Indeed, our interviewees noted that some participants have refused health screening services because they believed that service providers were inexperienced and poorly-trained. Furthermore, inadequately-trained service providers and poor service design could lead to participant complaints and reduce the willingness to participate again.

A lack of information was also identified as an important barrier to healthcare screening. Interviewees reported that most participants did not continue to take advantage of this service because they did not receive a notice to revisit. As a result, service providers tried various other ways to remind people to return for additional health screenings. Our results showed that all types of reminders, i.e., both letters and phone calls (including messages left on answering machines), were useful, with phone calls being the most useful [16]. However, these types of reminders were still far from perfect. For example, service providers frequently had difficulties connecting with seniors who live in rural areas because these seniors had little money and often no access to a phone. This illustrates that service providers need to consider communication barriers when dealing with seniors.

According to interviewees, many participants have noted that the registration system is a critical issue which reduces their willingness to undergo health screening and also impacts service quality. Other problems include variable access to screening services and screening equipment. In addition, people who live in urban areas and people who live in rural areas often suffer from different health problems. For example, rural populations 1) are more likely to experience higher rates of chronic conditions [17] and 2) tend to have less access to health information. Conversely, people who live in urban areas tend to have a higher risk of cancer and cardiovascular disease. Therefore, health services which are offered in community-based settings should be tailored to meet the needs of the communities they serve. 
Moreover, culturally-appropriate education materials are needed to increase awareness of how regular screenings can prevent chronic diseases. Community education campaigns could be useful. Educational strategies must also ensure that service providers are sensitized to historical and systemic barriers to health screening services, such as lower levels of education (including illiteracy). Sometimes communicating with participants and/or explaining how they should follow service procedures can be difficult. Finally, people sometimes feel too ashamed to take advantage of health services, which can cause them to ignore health problems.

In designing and enhancing health services, service gaps should be addressed and consideration should be given to how services are delivered. The first priority should be to elicit and understand a range of stakeholder perspectives on these issues. In this context, stakeholders include service providers, service supporters (such as the village representative in this study), and participants. On the other hand, in the current research, we found that it is necessary for rural communities to develop innovative ways to make healthcare more accessible to residents, for example, many of our research findings are especially valuable for the provision of healthcare services to elderly people in rural areas. These findings can help provide a deeper understanding of the issues that these individuals face in order to develop a successful, urgently-needed model of rural health service delivery.

A limitation of this study was that we only interviewed a small number of service providers who worked at the same community medical research center. Moreover, the perceptions and experiences of interviewees could be different from those of participants as they were in the same service chain. We recommend that future research pay greater attention to participant experiences.

The current research has identified factors that influence the willingness of participants. As such, the findings of this study will help to raise awareness of factors that influence how healthcare providers promote health services. The study highlighted that a combination of the attitudes of stakeholders, healthcare resources and sociodemographic can affect health-seeking behavior.

In order to provide effective care to participants of different cultural backgrounds, service providers should be encouraged to take into account that any service design process such as fair registration process arrangement and the limitations of rural populations to taking advantage of health service.

One of the strengths of this study was that the interviewees were of both genders and had extensive experience working in health screening services. Indeed, interviewees in this study were all first-line service providers; therefore, the study leads to true participants' service needs of health screening for chronic diseases. These findings could serve as valuable references for the development and improvement of preventive healthcare services.

Despite the fact that service providers often feel overwhelmed, they remain willing to work in health services if problems of labor shortages and inadequate employee training are addressed. In addition, to respect and support the contri- 
butions of service providers, the use of financial incentives and other incentives should be explored. This may also reduce the burnout of service providers and increase work efficiency in health service fields. Finally, changes in service should be evaluated from the perspectives of both service providers and participants.

\section{Conclusions}

Most of the interviewees in this study acknowledged the importance of detecting chronic diseases early. However, interviewees also reported that current screening services are not good enough to address service needs. These factors affect patient willingness to undergo health screening.

The findings of this study help elucidate which factors are likely to be effective in increasing screening rates for chronic diseases. Interview results confirm that health service providers are partly responsible for the provision of public health services and that screening remains the best option to reduce chronic disease mortality.

\section{Acknowledgements}

We would like to thank the 10 interviewees who participated in this study, sharing their opinions as well as their extensive work experience and knowledge with us. This research was supported by the "Academic Exchange and Cooperation Project" between the Top University Strategic Alliance (Taiwan) and the University of California, Berkeley (U.S.A.).

\section{Conflicts of Interest}

The authors declare no conflicts of interest regarding the publication of this paper.

\section{References}

[1] Raghupathi, W. and Raghupathi, V. (2018) An Empirical Study of Chronic Diseases in the United States: A Visual Analytics Approach to Public Health. International Journal of Environmental Research and Public Health, 15, 431. https://doi.org/10.3390/ijerph15030431

[2] Bauer, U.E., Briss, P.A., Goodman, R.A., Bowman, B.A. (2014) Prevention of Chronic Disease in the 21st Century: Elimination of the Leading Preventable Causes of Premature Death and Disability in the USA. The Lancet, 384, 45-42. https://doi.org/10.1016/S0140-6736(14)60648-6

[3] Centers for Disease Control and Prevention (CDC) (2012) Chronic Disease Prevention and Health Promotion.

[4] Anderson, G. and Horvath, J. (2004) The Growing Burden of Chronic Disease in America. Public Health Report, 119, 263-270. https://doi.org/10.1016/j.phr.2004.04.005

[5] Meetoo, D. (2008) Chronic Diseases. The Silent Global Epidemic. British Journal of Nursing, 17, 1320-1325. https://doi.org/10.12968/bjon.2008.17.21.31731

[6] Halpin, H.A., Morales-Suarez-Varela, M.M. and Martin-Moreno, J.M. (2010) Chronic 
Disease Prevention and the New Public Health. Public Health. Reviews, 32, 120-154. https://doi.org/10.1007/BF03391595

[7] World Health Organization (2005) Preventing Chronic Diseases: A Vital Investment. WHO Global Report, Geneva.

[8] Mosadeghrad, A.M. (2013) Healthcare Service Quality: Towards a Broad Definition. International Journal of Health Care Quality Assurance, 26, 203-219. https://doi.org/10.1108/09526861311311409

[9] Jensen, L.F., Pedersen, A.F., Andersen, B., Vestergaard, M., Vedsted, P. (2015) Non-Participation in Breast Cancer Screening for Women with Chronic Diseases and Multimorbidity: A Population-Based Cohort Study. BMC Cancer, 15, 798. https://doi.org/10.1186/s12885-015-1829-1

[10] Foley, G. and Timonen, V. (2015) Using Grounded Theory Method to Capture and Analyze Health Care Experiences. Health Services Research, 50, 1195-1210. https://doi.org/10.1111/1475-6773.12275

[11] Matsuzuki, H., Haruyama, Y., Muto, T., et al. (2013) Workers' Load and Job-Related Stress after a Reform and Work System Change in a Hospital Kitchen in Japan. Environmental Health and Preventive Medicine, 18, 171-176.

https://doi.org/10.1007/s12199-012-0291-9

[12] Hombergh, P.V.D., Kunzi, B., Elwyn, G., Doremalen, J.V., Akkermans, R., Grol, R. and Wensing, M. (2009) High Workload and Job Stress Are Associated with Lower Practice Performance in General Practice: An Observational Study in 239 General Practices in the Netherlands. BMC Health Services Research, 9, 118.

https://doi.org/10.1186/1472-6963-9-118

[13] Aninanya, G.A., Howard, N., Williams, J.E., Apam, B., Prytherch, H., Loukanova, S., Kamara, E.K. and Otupiri, E. (2016) Can Performance-Based Incentives Improve Motivation of Nurses and Midwives in Primary Facilities in Northern Ghana? A Quasi-Experimental Study. Global Health Action, 9, 1. https://doi.org/10.3402/gha.v9.32404

[14] McLaughlin, C.P. and Kaluzny, A.D. (2006) Continuous Quality Improvement in Health Care. 3rd Edition, Jones \& Bartlett, Sudbury, MA.

[15] Mosadeghrad, A.M. (2012) A Conceptual Framework for Quality of Care. Materia Socio-Medica, 24, 251-261. https://doi.org/10.5455/msm.2012.24.251-261

[16] McLean, S.M., Booth, A., Gee, M., Salway, S., Cobb, M., Bhanbhro, S., et al. (2016) Appointment Reminder Systems Are Effective but Not Optimal: Results of A Systematic Review and Evidence Synthesis Employing Realist Principles. Patient Preference and Adherence, 2016, 479-499. https://doi.org/10.2147/PPA.S93046

[17] Cheng, L., Tan, L., Zhang, L., Wei, S., Liu, L., et al. (2013) Chronic Disease Mortality in Rural and Urban Residents in Hubei Province, China, 2008-2010. BMC Public Health, 13, 713. https://doi.org/10.1186/1471-2458-13-713 